\title{
Le futur de la tradition : quelle muséologie pour les musées nationaux du Pacifique Sud?
}

The future of tradition : which museology for the national museum of South Pacific?

\section{Marion Bertin}

\section{CpenEdition}

\section{Journals}

Édition électronique

URL : http://journals.openedition.org/iss/2016

DOI : 10.4000/iss.2016

ISSN : 2306-4161

\section{Éditeur}

ICOM - International Council of Museums

\section{Édition imprimée}

Date de publication : 1 août 2020

Pagination : 95-108

ISBN : 978-2-491997-11-3

ISSN : 2309-1290

\section{Référence électronique}

Marion Bertin, «Le futur de la tradition : quelle muséologie pour les musées nationaux du Pacifique Sud? ", ICOFOM Study Series [En ligne], 48-1 | 2020, mis en ligne le 01 août 2020, consulté le 08 août 2020. URL : http://journals.openedition.org/iss/2016 ; DOI : https://doi.org/10.4000/iss.2016 


\section{Le futur de la tradition : quelle muséologie pour les musées nationaux du Pacifique Sud?}

\section{Marion Bertin}

École du Louvre \& Université de La Rochelle France

The future of tradition: which museology for the national museum of South Pacific?

Be they former colonial museums or newly-created establishments, national museums in the South Pacific islands play an important role in preserving, representing and affirming communities' cultures within these young states. The Western concept of the museum is adapted to the local environment with specific functions and relationships to collections. These national museums also question the representation of Oceanian societies, the diversity of communities who live there, their relationships and their histories.

Keywords: Pacific islands, museum, communities, traditions, nation, kastom. 


\section{RÉSUMÉ :}

Anciens musées coloniaux ou établissements nouvellement créés, les musées nationaux dans les îles du Pacifique Sud jouent un rôle important pour la conservation, la représentation et l'affirmation culturelle de ces jeunes États et de leurs communautés. Le concept occidental de musée s'y adapte localement, avec des missions et rapports aux collections spécifiques. Ces musées nationaux questionnent également les possibilités de représenter fidèlement les sociétés océaniennes, les diverses communautés qui y vivent, leurs relations et leur histoire.

Mots-clés : îles du Pacifique, musée, communautés, traditions, nation, kastom.

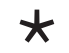

Le XIX ${ }^{e}$ siècle marque l'implantation des grandes puissances européennes dans le Pacifique Sud et la création de colonies. Dès cette époque, sont créés des musées défendant des buts économiques et politiques et jouant le rôle d'outils d'affirmation impérialiste et de domination (McLoed, 1998 ; Kasarhérou, 2003, p. 6I). En parallèle des expositions coloniales, dont la première se tient à Melbourne en I866, les musées coloniaux servent avant tout à mettre en valeur les ressources naturelles locales, qui sont autant de potentiels débouchés commerciaux. Quelques objets et productions rendant compte de la vie des populations insulaires intègrent les vitrines à titre plutôt anecdotique alors que les modes de vie se transforment sous l'effet de l'installation occidentale. Dans les années I970, au moment de l'accès à l'indépendance des archipels océaniens, culture, patrimoine et traditions anciennes jouent un rôle important dans l'affirmation identitaire et politique des populations autochtones (Eoe \& Swadling, I99I ; Babadzan, 2009). L'organisation de festivals et la création de centres culturels locaux, conçus par et pour les populations du Pacifique (Danielsson, 1980), révèlent des cultures vivantes et dynamiques, les réaffirmant, à l'encontre des musées coloniaux, plutôt fréquentés par des administrateurs et des touristes occidentaux (Geismar \& Tilley, 2003). Les anciens musées coloniaux sont toutefois maintenus et deviennent des lieux nationaux, repensés et rénovés (Bertin, 20rgb) tandis que, dans d'autres îles, de nouveaux établissements sont créés avec la même fonction'.

Les liens entre culture et affirmation politique dans les îles du Pacifique ont été abordés de nombreuses fois (Babadzan, r999a ; Babadzan, 1999b ; Babadzan,

I. Toutes les îles ne se dotent toutefois pas de musée, à l'exemple du Royaume de Tonga qui refuse longtemps d'en édifier un (Kasarhérou, 2003). 
2009; Foster, I995; Otto \& Thomas, 1997), mettant en exergue « le spectacle de la culture » (Babadzan, 2009); pourtant la place des musées dans cette relation reste peu étudiée, de même que les institutions muséales océaniennes elles-mêmes. Seuls quelques articles (Danielsson, I980 ; Hunt, 1978; McLeod, 1996; McLeod, 1998 ; Mead, 1983 ) et recueils de textes déjà anciens (Eoe \& Swadling, I99I ; Healy \& Witcomb, 2006 ; Stanley, 2007) permettent de donner un panorama des musées dans le Pacifique Sud. Ces institutions jouent néanmoins un rôle politique important afin d'établir une culture et une identité nationales (Kaeppler, I994), qu'ils soient implantés dans des petits États insulaires récemment indépendants ou des grands États parmi les premières puissances économiques mondiales, où demeure parfois une forme de colonisation interne. En prise avec le passé et le présent (Charles Hunt, 1978, p. 69), les musées nationaux sont des institutions cruciales pour comprendre les sociétés et États dans lesquels ils s'intègrent (Poulot, 20I6). Quelles sont les missions d'un musée national dans le Pacifique à l'ère post-coloniale, alors que plusieurs établissements connaissent des travaux et rénovations? Quelles cultures et quelles identités y sont représentées et exposées? Cet article montre quelques tendances générales sur l'adaptation d'un concept occidental et l'émergence d'une muséologie propre aux îles du Pacifique Sud, mêlant traditions océaniennes et missions fondatrices du musée.

\section{Tradition occidentale et traditions océaniennes}

Comme le souligne Emmanuel Kasarhérou, ancien directeur du musée de Nouvelle-Calédonie (MNC) et de l'Agence de Développement de la Culture kanak (ADCK), tous deux à Nouméa (Nouvelle-Calédonie), les musées dans le Pacifique se présentent comme un «héritage colonial à assumer » (Kasarhérou, 2003, p. 6I). Concept exogène, imposé localement (Bertin, 2019b ; Mc Loed, 1996), le musée est porteur et symbolique d'histoires douloureuses (Kasarhérou, 2003, p. 62). Toutefois, un article fondateur signé par l'anthropologue māori Sidney Moko Mead et publié en 1983 permet de rapprocher le musée d'autres lieux majeurs pour la vie sociale et rituelle des populations du Pacifique, les whare-whakairo māori ainsi que les maisons des hommes aux îles Salomon, qui présentent des fonctions relativement similaires.

Nous nous intéresserons plutôt aux fonctions fondamentales $d u$ musée qui consistent à entreposer des objets précieux et à les présenter à des personnes autres que celles qui les ont produites, le musée jouant ainsi un rôle utile en raison de ce quil contient et de la manière dont il est utilisé. (Mead, 1983, p. 98).

\section{9}

Dans cet article, Mead propose également quelques idées pour un modèle de musée autochtone dans le Pacifique, afin de mettre en lumière l'importance 
de la tradition orale et les liens que le musée entretient avec le «tapu $»^{\mathrm{T}}$, tant par les objets qu'il préserve que son bâtiment lui-même (Mead, I983, p. IOI). Il conseille également d' "intégrer [ces musées] au mode de vie traditionnel de la population » (Mead, I983, p. IOI), pour en faire des institutions pertinentes.

Parallèlement, les années 1970 et 1980 voient l'émergence d'un mouvement identitaire des populations autochtones du Pacifique incarné par des demandes d'accès à l'indépendance et d'affirmation culturelle autour de pratiques et coutumes anciennes réintroduites et remises en valeur (Babadzan, I999a). En Mélanésie, ce mouvement est reflété par le développement de la kastom, qui inclut un ensemble de traditions anciennes réifiées en tant que symboles nationaux, en en limitant les particularités locales (Babadzan, 2009; Bertin, 2019b ; Huffman, 2013; Geismar \& Tilley, 2003). Les festivals, organisés à l'échelle locale ou pan-océanienne, jouent un rôle majeur pour la promotion et la stimulation des pratiques culturelles (Babadzan, 20og). Dans les États nouvellement indépendants, les musées deviennent aussi des outils politiques, aidant à la « construction d'une identité post-coloniale nationale ${ }^{2}$ (Stanley, 2007, p. 7), autour d'une fierté culturelle. Dans d'autres États, ils permettent de répondre à l'agenda politico-social de réconciliation des diverses communautés pour un meilleur vivre-ensemble autour de valeurs communes.

\section{Des missions restées classiques}

La forme traditionnelle du musée, telle que pensée et entendue par le Conseil international des musées (ICOM), reste aujourd'hui valable : les missions du musée établies par la définition de 2007 sont proches de celles développées dans les institutions nationales du Pacifique 3 . Ainsi, ces musées ont des collections, les préservent, les transmettent, les exposent au public, les étudient (Paulias, I99I). L'inscription dans la durée est également présente. À titre d'exemple, les musées nationaux de la République des Palaos, archipel de Micronésie indépendant depuis I994, et des Îles Cook, archipel polynésien librement associé à Aotearoa - Nouvelle-Zélande depuis 1965, se définissent comme tels :

Le musée national de Belau (BNM) est une institution dynamique en faveur de la préservation et de la promotion du patrimoine national, de l'exposition des biens naturels, culturels, sociaux et historiques et du développement des arts à tous niveaux. Le musée national de Belau est une institution de recherche distinguée pour collecter,

\footnotetext{
I. Le tapu, ou tabu, désigne un ensemble de règles dictant l'accès à des savoirs, à des objets, à des personnes.

2. «The construction of a post-colonial national identity (traduction personnelle).

3. «Le musée est une institution permanente sans but lucratif, au service de la société et de son développement, ouverte au public, qui acquiert, conserve, étudie, expose et transmet le patrimoine matériel et immatériel de l'humanité et de son environnement à des fins d'études, d'éducation et de délectation. »
} 
préserver et présenter les objets à valeur scientifique, culturelle, historique et esthétique. Le but du musée est de préserver et protéger le patrimoine culturel national par le biais de la collection, l'identification, la documentation, la préservation, l'interprétation et l'exposition de spécimens, d'artefacts et autres possessions culturelles des Palaos ${ }^{1}$ (Belau National Museum, s.d.).

Le musée national des îles Cook ouvre le 14 octobre 1992 en tant qu'institution pour présenter, éduquer et préserver l'histoire culturelle des îles Cook. Nous ne sommes pas spécifiquement un musée d'art, d'anthropologie, d'ethnologie, de biologie marine ou d'histoire naturelle, mais un musée avec des descriptions générales entourant notre culture matérielle et visuelle ${ }^{2}$ (Ministry of Cultural Development, s.d.).

Des fonctions d'archives et de bibliothèques nationales sont également souvent conjointes aux musées. Une organisation interne en différents services aux fonctions et missions distinctes est établie, ainsi qu'une structure hiérarchique. Les tutelles varient, qu'elles soient liées au développement culturel (îles Cook), à la Culture, la Condition féminine et la Citoyenneté (Nouvelle-Calédonie) ou aux Arts, Culture et Patrimoine (Aotearoa - Nouvelle-Zélande). La garantie de protection et de conservation est fondamentale pour les musées qui sont en lien avec les autorités coutumières et les douanes pour contrôler les trafics illicites et les exportations illégales d'objets hors des territoires, en témoignent la Nouvelle-Calédonie, la Papouasie-Nouvelle-Guinée ou le Vanuatu par exemple.

Toutefois, le rôle de conservatoire n'est pas le seul. Une des particularités des musées du Pacifique consiste en l'apport du modèle du centre culturel, dont plusieurs se développent à partir des années I970, comme outil d'affirmation et de représentation politique et culturelle (Danielsson, I980). À une échelle plus réduite en lien avec les communautés locales, ces centres culturels s'in-

\footnotetext{
I. "The Belau National Museum (BNM) is a dynamic institution for the preservation and promotion of the national heritage, exhibition of natural, cultural, social and historical values, and the development of arts at all levels. The Belau National Museum is an institution of learning established to collect, preserve and display object of scientific, cultural, historical and aesthetic value. The Museum's purpose is to preserve and protect the nation's cultural heritage through collection, identification, documentation, preservation, interpretation and exhibition of specimens, artifacts and other Palauan cultural property. » (traduction personnelle).

2. "The Cook Islands National Museum was opened on October I4th 1992 as an institution to display, educate and preserve the cultural history of the Cook Islands. We are not specifically a Museum of Art, Anthropology, Ethnology, Marine Biology or Natural History, but it is a museum with general descriptions encompassing our visual and material culture. » (traduction personnelle).
} 
téressent en premier lieu à la documentation et la conservation des savoirs et des techniques, afin d'assurer leur transmission. L'intérêt de tels établissement se porte ainsi plus volontiers vers le patrimoine immatériel que sur ses formes matérielles. Les productions contemporaines y sont également valorisées. Les frontières sont à présent parfois poreuses entre centre culturel et institution muséale (Danielsson, I980; Stanley, 2007) : des programmes de recherches et de collectes des savoirs et traditions immatériels sont engagés en ce sens par les musées, qui permettent ensuite une programmation mettant en évidence des cultures dynamiques à travers danses, visites guidées et commentées par des tenants des savoirs ou préparation de plats culinaires. Ces activités sont complémentaires des espaces d'exposition qui donnent à voir une vision large de chaque archipel dans leurs aspects historiques, anthropologiques, archéologiques, naturels et artistiques. Des œuvres récentes produites par des artistes locaux côtoient des productions plus anciennes avec lesquelles elles dialoguent et s'inscrivent dans un temps long, dispositif de présentation employé au musée de Nouvelle-Calédonie (MNC) à Nouméa ou au Papua New Guinea National Museum and Art Gallery (PNGNMAG) de Port Moresby.

Le rôle social du musée et sa mission de protection des collections en faveur des communautés dont elles sont originaires sont toutefois plus forts qu'ailleurs. Pour le code de déontologie rédigé en 2006 par l'Association des musées des îles du Pacifique (PIMA), les musées et centres culturels :

I. sont les gardiens des collections de ressources culturelles dont ils ont la responsabilité, principalement pour les communautés qui les ont créées et les populations du Pacifique, deuxièmement pour l'intérêt des autres populations et communautés ;

2. ont une responsabilité essentielle pour assister les communautés dans le maintien et la sauvegarde de leur patrimoine culturel immatériel en cours ;

3. construire et maintenir des relations de compréhension culturelle et de respect mutuel avec les communautés qu'ils servent (Pacific Islands Museums Association, 2006)'.

Les musées sont ainsi à la fois des gardiens des savoirs ancestraux et des lieux pour les faire (re)connaitre (Mead, 1983).

\footnotetext{
I. « Pacific Islands museums and cultural centres:

I. are the custodians of collections of cultural resources that they hold in trust, foremost for their creator communities and the peoples of the Pacific, secondly for the benefit of people and communities elsewhere;

2. have a primary responsibility to assist communities to maintain and safeguard their continuing intangible cultural heritage;

3. build and maintain relations of cultural understanding and mutual respect with the communities they serve. » (traduction personnelle).
} 


\section{Changer l'enveloppe, changer les pratiques}

Quelques modifications dans la physionomie du musée, son organisation interne ainsi que la gestion des collections permettent de lui donner une résonance véritablement océanienne ${ }^{\mathrm{I}}$. En premier lieu, l'architecture des établissements les plus récents s'éloigne du modèle européen pour prendre exemple sur les constructions locales (McLoed, I996, p. 276), ce qui est le cas du bâtiment du Vanuatu Kaljoral Senta (VKS) à Port-Vila (Vanuatu) ouvert au public en I995 ou du Salomon Islands National Museum à Honiara (îles Salomon). Les travaux du MNC à Nouméa visent à rénover le bâtiment de style Art Déco et à lui donner l'aspect d'une peau de serpent, à base de bois et matériaux locaux, l'ancrant plus dans son territoire («Du musée de Nouvelle-Calédonie au MUZ », 20I8). L'exposition ne s'arrête pas aux murs du musée, et les jardins et espaces extérieurs ont aussi leur importance : plusieurs musées intègrent ainsi des reproductions de maisons et lieux de rassemblements sociaux coutumiers dans leurs jardins, comme au MNC ou au BNM à Koror. Ces espaces servent en outre aux programmations culturelles, qui dépassent une approche strictement matérielle, pour intégrer de nombreux rassemblements, fêtes et cérémonies (Foana'ota, I99I).

Un des changements majeurs réside dans les équipes en charge de la gestion des musées et des collections, qui ne sont plus seulement sous la responsabilité d'administrateurs coloniaux et d'expatriés. Le personnel inclut des membres des communautés autochtones (Kasaréhou, 2003; McLoed, r998), en charge de leurs propres pratiques culturelles. En plus de services de conservation matérielle des collections, des dépositaires des traditions et des savoirs portent un autre regard sur les objets. En Aotearoa - Nouvelle-Zélande, État fondé sur un bi-culturalisme māori et pakeha² depuis les années I980, le National Museum of New Zealand Te Papa Tongarewa (Te Papa) observe le Mana Taonga, principe reconnaissant une filiation et un droit des iwis māori sur les objets qui leur sont liés (McCarthy, 20II). L'équipe de conservateurs et de curators comprend des personnalités d'ascendance māori, chargées de maintenir et diffuser le Mātauranga Māori, le savoir māori, au sein de l'institution. De plus, un service du musée, le Ngā Manu Atarau, est spécifiquement lié aux communautés, aux restitutions d'objets et au développement culturel («Organisational structure », s.d.). Des responsables de collections dépositaires des savoirs et traditions autochtones sont aussi présents au Bishop Museum d'Honolulu (Hawaii), ainsi qu'au VKS. Au-delà de la conservation matérielle des objets, que Sidney Moko Mead souligne comme étant une pratique avant tout occidentale (I983, p. Ioo), la préservation et la transmission des savoirs et techniques de leur fabrication importent davantage. Le Cook Islands National Museum conserve ainsi de nombreuses répliques d'objets traditionnels de l'archipel (Ministry of Cultural

\footnotetext{
I. « Change or die » résume Soroi Marepo Eoe en r99I (Eoe, I99I).

2. «Pakeha » est le nom donné aux descendants de colons installés en Aotearoa - Nouvelle-Zélande.

3. Les iwi sont les unités familiales élargies chez les populations māori.
} 
Development, s.d.). Des boutiques peuvent proposer à la vente les répliques d'objets ainsi produites, au Belau National Museum (Morei, I99I, pp. 172-I73) par exemple, ce qui constitue une forme d'encouragement et de valorisation de la production contemporaine. La protection et la continuité des savoirs autochtones passent en outre par l'engagement de ces communautés : des programmes impliquant des fieldworkers bénévoles dans la collecte et la préservation de leurs propres pratiques culturelles, matérielles ou immatérielles, sont mis en place au VKS avec une importance accordée aux femmes (Bolton, 2003 ; Bolton, 2007) et au Salomon Islands National Museum où les jeunes générations tiennent une place majeure (Foana'ota, 2007).

L'inclusion des communautés autochtones permet in fine de repenser le rapport entretenu avec les objets des collections et leurs modalités de conservation. Les objets sacrés, dont la valeur peut alors être reconnue, sont particulièrement concernés (Bertin, 20I8 ; Bertin, 2019a). Des protocoles de gestion et d'exposition existent au VKS, où différentes réserves permettent de distinguer les niveaux de sacré des objets. Une « Tabu Room » permet de conserver les objets les plus sacrés et secrets de l'archipel du Vanuatu ainsi que les enregistrements de cérémonies par les fieldworkers qui en sont les dépositaires (Bertin, 2019a; Geismar, 2003). L'exposition tient aussi compte de ces prérogatives, au VKS comme au Te Papa ou au MNC où les membres du personnel familiers des pratiques culturelles autochtones peuvent porter un avis consultatif sur les modalités à privilégier ainsi que les objets à maintenir hors du regard. La diversité des membres du personnel, venant de différentes communautés, permet une exposition à la première personne.

\section{Un musée national pour qui ?}

Depuis plusieurs décennies, les musées du Pacifique «deviennent des espaces de négociation et de référence culturelle, de même que des dépôts de savoirs et d'objets $^{1} \gg$ (McLeod, 1996, p. 278). La place des communautés autochtones y est de plus en plus grande, qu'il s'agisse de leur représentation par les objets et les expositions, de leur implication et engagement à titre bénévole ou salarié des établissements ou encore de l'écoute de leurs préférences pour la protection et la gestion des collections. Le modèle muséal occidental est ainsi converti en un modèle autochtone (McLeod, I996, p. 278) : le PNGNMAG de Port Moresby, créé en 1977 au moment de l'indépendance et réouvert en octobre 2018 après des travaux de rénovation, est ainsi qualifié d'« authentique institution indigène ${ }^{2}$ » («Museum and art gallery reopened », 2018). «Agents pour promouvoir les cultures autochtones » (Stanley, 2007, p. I), les musées nationaux contribuent toutefois à une «sacralisation des passés culturels et des traditions » (Babadzan, I999a, p. 7). La mise en valeur d'une kastom privilégie certaines traditions,

\footnotetext{
I. « $[\ldots]$ becoming spaces of negotiation and cultural reference, as well as repositories of skills and objects. » (traduction personnelle).

2. «An authentically indigenous institution» (traduction personnelle).
} 
réinventées ou réinterprétées, principalement pré-coloniales (Babadzan, 1999b). Au VKS de Port-Vila comme au MNC de Nouméa, des sections présentent longuement les systèmes d'échanges entre clans et tribus pratiqués avant la colonisation européenne et restés importants pour comprendre les relations entretenues par les nombreuses communautés autochtones au Vanuatu et en Nouvelle-Calédonie. Le Museo antropológico del Padre Sebastián Englert (MAPSE) à Rapa Nui (île de Pâques) se concentre également principalement sur les cultures pascuanes anciennes, avec lesquelles des liens contemporains sont rétablis («Misión », s.d.). Les objets anciens acquis par des Occidentaux puis revenus sur leurs territoires d'origine, qu'ils s'agissent d'achats sur le marché de l'art («Un objet kanak rentre au pays », 20I8), de dons (« Kini Ke Kua. Transformative Images », 20I8) ou de demandes de retours («Repatriation Karanga Aotearoa », s.d.) tiennent également une place importante. Toutefois, les publics autochtones entretiennent un rapport paradoxal avec les collections des établissements muséaux. Une relation inversement proportionnelle se crée entre les communautés, les musées et leurs collections : la visite du musée n'intervient qu'après une prise de distance avec les objets exposé (Kasarhérou, 2003). La culture et les traditions sont vécues, des modalités auxquelles participer, dont faire l'expérience, et ne sauraient être regardées de loin (Hunt, 1978, p. 72).

Les questions de la représentation et de l'inclusion d'autres communautés occupent les débats dans de nombreux archipels. La complexité des sociétés du Pacifique est rarement reflétée et certaines communautés sont invisibilisées dans l'espace des musées nationaux. Les différents flux et arrivées de populations, en premier lieu desquels le colonialisme, ne sont pas unanimement évoqués. Les grands États du Pacifique, tels que l'Australie, Aotearoa - Nouvelle-Zélande, ou les collectivités d'outre-mer françaises - Nouvelle-Calédonie et Polynésie française - sont particulièrement concernés. Le bi-culturalisme māori et pakeha revendiqué par la Nouvelle-Zélande éclipse un multi-culturalisme et la présence d'autres communautés (McCarthy, 2ori, p. ro). En Nouvelle-Calédonie, le projet actuel de rénovation du MNC vise à une représentation plus fidèle de la société calédonienne, tandis que l'ancienne présentation est principalement dédiée aux sociétés kanak, dans l'esprit du «destin commun » politique et social ( Du musée de Nouvelle-Calédonie au MUZ », 20I8) et alors que les débats autour de l'indépendance sont omniprésents. D'autres États plus petits sont également confrontés à ces problématiques et défis, à l'instar de la République des Fidji : le Fiji Museum, créé pendant l'époque coloniale au début du XX siècle, connait des conflits quant à la représentation de la société fidjienne, concernant notamment les communautés indiennes, chinoises et polynésiennes arrivées par vagues d'émigration successives. «Dans une société multi-ethnique, essayer d'affirmer des identités multiples au sein d'une histoire nationale dans une institution nationale fait du musée un espace politiquement contestét (Ramsay, 2013, p. 190).

\footnotetext{
I. «In a multi-ethnic society, trying to assert multiple identities in a national history in a national institution made the museum a politically contested space. » (traduction personnelle).
} 
Les relations inter-communautaires sont un autre défi de représentation, en raison des nombreux conflits sous-jacents. Les rencontres et connexions sont davantage éclairées entre les différentes îles, afin de mettre en évidence l'histoire d'un peuplement initial commun des archipels comme c'est le cas au Bishop Museum d'Honolulu d'Hawaii ou au Te Papa (Christophe, 2016). Approcher une dimension historique fait également partie des difficultés des musées nationaux du Pacifique, en raison des divergences de points de vue (Healy, 2006). L'histoire coloniale (Losche, 2006) et la guerre du Pacifique sont ainsi difficilement abordées, étant pourtant majeures pour comprendre les sociétés contemporaines océaniennes.

\section{Conclusion : quel avenir pour les musées nationaux du Pacifique?}

Les musées nationaux du Pacifique sont particulièrement intéressants à étudier pour comprendre les enjeux et défis contemporains de ce type d'institutions. Outre les questions de représentations et de discours, les musées océaniens doivent faire face à d'autres interrogations quant à leur avenir. La fréquentation continue d'être une gageure afin que les musées ne soient pas seulement l'apanage des touristes de passage, mais réellement des lieux où les communautés locales se sentent toutes incluses et bienvenues. Face à la multiplication des demandes de retours d'objets conservés dans des collections muséales internationales, émanant notamment de Rapa Nui ou de Aotearoa - Nouvelle-Zélande, la future place qui pourra leur être accordée dans les musées nationaux et les discours qui les accompagneront posent déjà question. Les sources de financement sont également en jeu, quand les travaux entrepris au MNC sont permis par un contrat de développement entre l'État français et la Nouvelle-Calédonie à l'échéance 2017-202I ( (Du musée de Nouvelle-Calédonie au MUZ », 2018) ou que la rénovation du PNGNMAG a été permise grâce à des fonds provenant d'Australie («Museum and art gallery reopened », 2018), avec qui la Papouasie-Nouvelle-Guinée continue d'entretenir des relations fortes depuis son accès à l'indépendance en 1975 . Ces financements en partie extérieurs permettent finalement d'interroger la place des relations géopolitiques internationales à l'œuvre dans le Pacifique et la question du pouvoir qu'elles exercent sur les institutions nationales, à l'heure où plusieurs archipels souffrent de contextes politiques troublés. Les musées n'en sont qu'un maigre reflet, mais en permettent une image révélatrice. 


\section{Références}

About the Belau National Museum (s.d.). Belau National Museum. Page consultée le iz novembre 2019, à l'adresse $:$ http://belaunationalmuseum.net/ about.html

Badadzan, A. (1999a). Avant-propos. Culture, coutume, nation : les enjeux d'un débat. Journal de la Société des Océanistes, I09, 7-I2.

Badadzan, A. (1999b). L'Invention des traditions et le nationalisme. Journal de la Société des Océanistes, I09, 13-35.

Badadzan, A. (2009). Le Spectacle de la culture. Paris, France : L'Harmattan.

Bertin, M. (2018). Tapu et musée : conserver et exposer des objets océaniens. Dans F. Mairesse (Dir.), Museology and the sacred (pp. 39-43). Paris, France : ICOFOM.

Bertin, M. (2019a). Allier kastom et tabu au musée : gestion et exposition des objets du Vanuatu. ICOFOM Study Series, 47, pp. 4I-56.

Bertin, M. (20I9b). Décoloniser les musées du Pacifique : quelques défis pour le futur. Dans K. Smeds (Dir.), The Future of tradition in museology. Materials for a discussion (pp. 22-26). Paris, France : ICOFOM.

Bolton, L. (2003). Unfolding the moon: enacting women's kastom in Vanuatu. Honolulu, États-Unis : University of Hawai'i Press.

Bolton, L. (2007). Resourcing change: fieldwork, the Women's Cultural Project and the Vanuatu Cultural Centre. Dans Stanley, N. (Dir.). The Future of Indigenous Museums: Perspectives from the Southwest Pacific (pp. 23-37). Londres, Grande-Bretagne : Berghan Books.

Christophe, A. (2016). Exhibiting connections, connecting exhibitions: Constructing trans-Pacific relationships though museum displays in Oceania (2006-2016). (thèse de philosophie). Norwich, Grande-Bretagne : University of East Anglia.

Danielsson, B. (1980). Cultural Centers in Oceania. Pacific Perspective, 9, I, I3-20.

Du musée de Nouvelle-Calédonie au MUZ ». (I3 juillet 20I8). Musée de Nouvelle-Calédonie. Page consultée le 7 mars 20r9, à l'adresse : https:// museenouvellecaledonie.nc/actualites/thematique/le-musee/du-museede-nouvelle-caledonie-au-muz

Eoe, S. M., \& Swadling, P. (Dir.) (199I). Museums and cultural centres in the Pacific. Port Moresby, Papouasie-Nouvelle-Guinée : Papua New Guinea National Museum.

Foana'ota, L. (1991). The Solomon islands national museum. Dans Eoe, S. M., \& Swadling, P. (Dir.). Museums and cultural centres in the Pacific (pp. Io7- 
II2). Port Moresby, Papouasie-Nouvelle-Guinée : Papua New Guinea National Museum.

Foana'ota, L. (2007). The future of indigenous museums: the Solomon islands case. Dans Stanley, N. (Dir.). The Future of Indigenous Museums: Perspectives from the Southwest Pacific (pp. 38-46). Londres, Grande-Bretagne : Berghan Books.

Foster, R. J. (Dir.). Nation Making. Emergent identities in postcolonial Melanesia. Ann Arbor, États-Unis : University of Michigan Press, I995.

Geismar, H., Tilley, C. (2003). Negociating Materiality: international and local museums practices at the Vanuatu Cultural Centre and National Museum. Oceania, 73, I70-188.

Healy, C. (2006). Experiments in culture: an introduction. Dans Healy, C., \& Witcomb, A. (Dir.). South Pacific Museums. Experiments in culture (pp. 9-I5). Victoria, Australie : Monash University.

Huffman, K. W. (2013). 'Noho'n'dou yene nieve nungute I numuwo' hyene' - Respect is the foundation of life. Dans Howarth, C. (Dir.). Kastom. Art of Vamuatu (pp. 30-35). Coogee, Australia : New South Books.

Hunt, C. (I978). Museums in the Pacific islands: a metaphysical justification. Museum, XXX, 2, 69-75.

Kaeppler, A. (I994). Paradise regained: The role of Pacific museums in forging national identity. Dans Kaplan, F. (Dir.). Museums and the making of ourselves. Londres, Grande-Bretagne \& New York, États-Unis : Leicester University Press.

Kasarhérou, E. (2003). Musées et populations autochtones en Océanie. Dans Réseaux autochtones, partenariats, questions d'éthique (pp. 59-65). Lyon, France : Muséum d'histoire naturelle.

Kini Ke Kua. Transformative Images » (2018). Bishop Museum. Page consultée le I3 novembre 2019, à l'adresse : https://www.bishopmuseum.org/kini-ke-kua/

Losche, D. (2006). Hiroshima mon amour. Representation and violence in new museums of the Pacific. Dans Healy, C., \& Witcomb, A. (Dir.). South Pacific Museums. Experiments in culture (pp. 235-249). Victoria, Australie : Monash University.

McCarthy, C. (20II). Museums and Maōri. Heritage professionals, indigenous collections, current practice. Wellington, Aotearoa - Nouvelle-Zélande : Te Papa Press.

McLeod, R. (I996). Museum in the Pacific: Reflections on an "introduced concept" in transition. Sciences et développement, 5, 275-280.

McLeod, R. (I998). Postcolonialism and museum knowledge: revisiting the museums of the Pacific. Pacific Sciences, 52, 4, 308-3i 8. 
Mead, S. M. (1983). Modèles autochtones en Océanie. Museum, 34, 2, 98-гог.

Misión (s.d.). Museo Rapa Nui. Page consultée le I3 novembre 20I9, à l'adresse : https://www.museorapanui.gob.cl/sitio/Secciones/Quienes-Somos/ Mision/

Morei, O. E. (199I). The Belau National Museum. Dans Eoe, S. M., \& Swadling, P. (Dir.). Museums and cultural centres in the Pacific (pp. I70-173). Port Moresby, Papouasie-Nouvelle-Guinée : Papua New Guinea National Museum.

Museum and art gallery reopened (I5 octobre 2018). Post-Courier. Page consultée le I3 novembre 2019, à l'adresse : https:/postcourier.com.pg/ museum-art-gallery-reopened/

National Museum (s.d.). Ministry of Cultural Development. Page consultée le i3 novembre 2019, à l'adresse : https://www.culture.gov.ck/national-museum/

Organisational structure » (s.d.). Te Papa. Page consultée le i3 novembre 2019, à l'adresse : https://www.tepapa.govt.nz/about/what-we-do/organisation-structure

Otto, T., \& Thomas, N. (Dir.). Narratives of nation in the South Pacific. Amsterdam, Pays-Bas: Harwood Academic publishers, 1997.

Pacific Islands Museums Association (2006). PIMA Code of Ethics for Pacific Museums and Cultural Centres. Page consultée le iz novembre 2019, à l'adresse : https://www.wipo.int/export/sites/www/tk/en/databases/ creative heritage/docs/pima code ethics.pdf

Paulias, N. E. (I99I). The cultural heritage in the Pacific: preservation, development and promotion. Dans Eoe, S. M., \& Swadling, P. (Dir.). Museums and cultural centres in the Pacific (pp. 5-I4). Port Moresby, Papouasie-Nouvelle-Guinée : Papua New Guinea National Museum.

Poulot, D. (2016). Introduction. Le musée et le politique. Culture \& Musées, $\mathrm{n}^{\circ} 28,2016$, pp. 13-29.

Ramsay, A. (2013). Challenges to a house of treasures: cultural history of the Fiji museum. (Master degree). Suva, Fidji : University of the South Pacific.

Repatriation Karanga Aotearoa » (s.d.). Te Papa. Page consultée le iz novembre 2019, à l'adresse : https://www.tepapa.govt.nz/about/repatriation

Stanley, N. (2007). Indigeneity and museum practice in the Southwest Pacific. Dans Stanley, N. (Dir.). The Future of Indigenous Museums: Perspectives from the Southwest Pacific (pp. I-20). Londres, Grande-Bretagne : Berghan Books. 
Papers - Le futur de la tradition [...]

Un objet kanak rentre au pays (2 mars 20r8). Gouvernement de Nouvelle-Calédonie. Page consultée le iz novembre 2019, à l'adresse : https://gouv.nc/ actualites/o2-03-20I8/un-objet-kanak-rentre-au-pays 